\title{
EQUIDISTRIBUTION OF EXPANDING TRANSLATES OF CURVES AND DIRICHLET'S THEOREM ON DIOPHANTINE APPROXIMATION
}

\author{
NIMISH A. SHAH
}

\begin{abstract}
We show that for almost all points on any analytic curve on $\mathbb{R}^{k}$ which is not contained in a proper affine subspace, the Dirichlet's theorem on simultaneous approximation, as well as its dual result for simultaneous approximation of linear forms, cannot be improved. The result is obtained by proving asymptotic equidistribution of evolution of a curve on a strongly unstable leaf under certain partially hyperbolic flow on the space of unimodular lattices in $\mathbb{R}^{k+1}$. The proof involves ergodic properties of unipotent flows on homogeneous spaces.
\end{abstract}

\section{IntroduCtion}

The Dirichlet's theorem on simultaneous approximation of any $k$ real numbers $\xi_{1}, \ldots, \xi_{k}$ says the following:

(A) For any positive integer $N$ there exist integers $q_{1}, \ldots, q_{k}, p$ such that

$$
\left|q_{1} \xi_{1}+\cdots+q_{k} \xi_{k}-p\right| \leq N^{-k} \quad \text { and } \quad 0<\max _{1 \leq i \leq k}\left|q_{i}\right| \leq N
$$

(B) For any positive integer $N$ there exist integers $q, p_{1}, \ldots, p_{k}$ such that

$$
\left|q \xi_{i}-p_{i}\right| \leq N^{-1} \quad \text { and } \quad \max _{1 \leq i \leq k}|q| \leq N^{k} .
$$

After [6], we say that the D.Th. (A) (respectively, (B)) cannot be improved for $\boldsymbol{\xi}=\left(\xi_{1}, \ldots, \xi_{k}\right) \in \mathbb{R}^{k}$ if for any $0<\mu<1$ the following holds:

$(\mathrm{A} \mu)$ There are infinitely many positive integers $N$ for which the pair of inequalities

$$
\left|\left(q_{1} \xi_{1}+\cdots+q_{k} \xi_{k}\right)-p\right| \leq \mu N^{-k} \quad \text { and } \quad 0<\max _{1 \leq i \leq k}\left|q_{i}\right| \leq \mu N
$$

are insoluble in integers $q_{1}, \ldots, q_{k}, p$ (respectively,

2000 Mathematics Subject Classification. 22E40, 11J83. 
$(\mathrm{B} \mu)$ there are infinitely many positive integers $N$ for which the pair of inequalities

$$
\left|q \xi_{i}-p_{1}\right| \leq \mu N^{-1} \quad \text { and } \quad 0<|q| \leq \mu N^{k}
$$

are insoluble in integers $\left.q, p_{1}, \ldots, p_{k}\right)$.

In [6], Davenport and Schmidt proved that D.Th. (A) and (B) cannot be improved for almost all $\boldsymbol{\xi} \in \mathbb{R}^{k}$.

One says that D.Th. (A) (respectively, (B)) cannot be $\mu$-improved for $\xi \in \mathbb{R}^{k}$ if $(\mathrm{A} \mu)$ (respectively, $\left.(\mathrm{B} \mu)\right)$ holds. In [7] Davenport and Schmidt showed that D.Th. (A) cannot be $(1 / 4)$-improved for the pair $\left(\xi, \xi^{2}\right)$ for almost all $\xi \in \mathbb{R}$. This result was generalized by Baker [1] for almost all points on 'smooth curves' in $\mathbb{R}^{2}$, by Dodson, Rynne and Vickers [8] for almost all points on 'higher dimensional curved submanifolds' of $\mathbb{R}^{k}$, and by Bugeaud [2] for almost all points on the curve $\left(\xi, \xi^{2}, \ldots, \xi^{k}\right)$; in each case $(\mathrm{A} \mu)$ holds for some small value of $\mu<1$ depending on the curvature of the smooth submanifold. Their proofs typically involve the technique of regular system introduced in [6].

Recently the problem was recast in the language of flows on homogeneous spaces by Kleinbock and Weiss [11] using observations due to Dani [3], as well as Kleinbock and Margulis [10]. In [11] it was shown that D.Th. (A) and (B), as well as its various generalizations, cannot be $\mu$-improved for almost all points on any non-degenerate curve on $\mathbb{R}^{k}$ for some small $\mu<1$ depending on the curve. In this article, we shall strengthen such results for all $0<\mu<1$ :

Theorem 1.1. Let $\varphi:[a, b] \rightarrow \mathbb{R}^{k}$ be an analytic curve such that its image is not contained in a proper affine subspace. Then Dirichlet's theorem (A) and (B) cannot be improved for $\varphi(s)$ for almost all $s \in$ $[a, b]$.

This result will be deduced from a result about limiting distributions of certain expanding sequence of curves on the space of lattices in $\mathbb{R}^{k+1}$. A refinement of Theorem 1.1 is obtained in Theorem 1.4.

1.0.1. Notation. Let $G=\mathrm{SL}(n, \mathbb{R}), n \geq 2$. For $t \in \mathbb{R}$ and $\boldsymbol{\xi}=$ $\left(\xi_{1}, \ldots, \xi_{n-1}\right) \in \mathbb{R}^{n-1}$, define

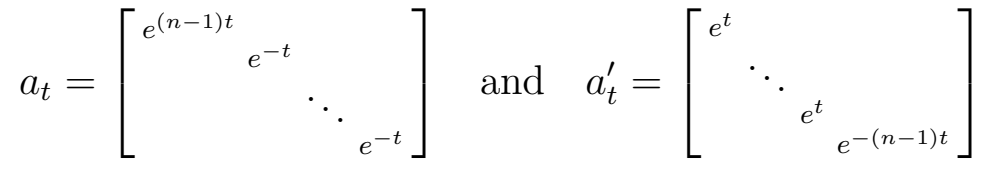

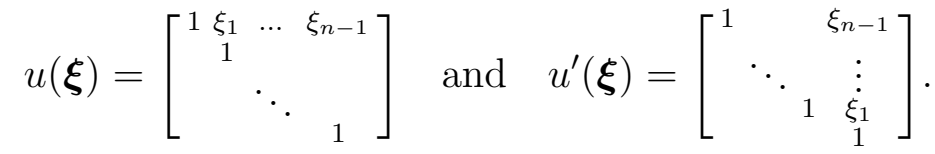

The main goal of this article is to prove the following: 
Theorem 1.2. Let $\varphi:[a, b] \rightarrow \mathbb{R}^{n-1}$ be an analytic curve whose image is not contained in a proper affine subspace. Let $\Gamma$ be a lattice in $G$. The for any $x_{0} \in G / \Gamma$ and any bounded continuous function $f$ on $G / \Gamma$,

$$
\lim _{t \rightarrow \infty} \frac{1}{|b-a|} \int_{a}^{b} f\left(a_{t} u(\varphi(s)) x_{0}\right) d s=\int_{G / \Gamma} f d \mu_{G},
$$

where $\mu_{G}$ is the $G$-invariant probability measure on $G / \Gamma$. Similarly,

$$
\lim _{t \rightarrow \infty} \frac{1}{|b-a|} \int_{a}^{b} f\left(a_{t}^{\prime} u^{\prime}(\varphi(s)) x_{0}\right) d s=\int_{G / \Gamma} f d \mu_{G} .
$$

This result corroborates [9, §4.3], [11, §4.1], and answers [17, Question 6.1]. In fact, we will prove the following more general statement.

Theorem 1.3. Let $L$ be a Lie group and $\Lambda$ a lattice in L. Let $\rho$ : $G \rightarrow L$ be a continuous homomorphism. Let $x_{0} \in L / \Lambda$ and $H$ be a minimal closed subgroup of $L$ containing $\rho(G)$ such that the orbit $H x_{0}$ is closed, and admits a finite $H$-invariant measure, say $\mu_{H}$. Then for any bounded continuous function $f$ on $L / \Lambda$ the following holds:

$$
\lim _{t \rightarrow \infty} \frac{1}{|b-a|} \int_{a}^{b} f\left(\rho\left(a_{t} u(\varphi(s))\right) x_{0}\right) d s=\int_{H x_{0}} f d \mu_{H} .
$$

The first part of Theorem 1.2 follows from Theorem 1.3 by taking $L=G, \rho$ the identity map, and $\Lambda=\Gamma$; in this case $H=G$.

Define an automorphism $\sigma: G \rightarrow G$ as follows: let $s_{k}$ be the permutation on the $n$-coordinates of $\mathbb{R}^{n}$ which exchanges the $i$-th and the $(n+1-i)$-th coordinates for $1 \leq i \leq n$, and we let $\sigma(g)=s_{k}\left({ }^{\mathbf{t}^{-1}}\right) s_{k}^{-1}$ for all $g \in G$. Then $\sigma\left(a_{t}\right)=a_{t}^{\prime}$ and $\sigma(u(\boldsymbol{\xi}))=u^{\prime}(\xi)$. Now the second part of Theorem 1.2 follows from Theorem 1.3 by taking $L=G, \rho=\sigma$ and $\Lambda=\Gamma$; we observe that $H=L$ in this case.

1.0.2. Next we take $L=G \times G, \Gamma=\operatorname{SL}(n, \mathbb{Z}), \Lambda=\Gamma \times \Gamma$ and $\rho(g)=(g, \sigma(g))$ for all $g \in G$. Note that $\sigma(\Gamma)=\Gamma$, and hence $\rho(\Gamma) \subset \Lambda$ and $\rho(\Gamma)$ is a lattice in $\rho(G)$. Therefore if we let $x_{0}=\Lambda$, then $\rho(G) x_{0}$ is closed and admits a finite $\rho(G)$-invariant measure; in other words, we have $H=\rho(G)$. Now using Theorem [1.3, in the next section we will deduce the following enhancement of Theorem 1.1

Theorem 1.4. Let $\varphi=\left(\varphi_{1}, \ldots, \varphi_{k}\right): I=[a, b] \rightarrow \mathbb{R}^{k}$ be an analytic curve whose image is not contained in a proper affine subspace. Let $\mathcal{N}$ be an infinite set of positive integers. Then for almost every $s \in I$ and any $\mu<1$, there exist infinitely many $N \in \mathcal{N}$ such that both the following pairs of inequalities are simultaneously insoluble:

$$
\left|q_{1} \varphi_{1}(s)+\cdots+q_{k} \varphi_{k}(s)-p\right| \leq \mu N^{-k} \quad \text { and } \quad \max _{1 \leq i \leq k}\left|q_{i}\right| \leq \mu N
$$


for $\left(q_{1}, \ldots, q_{k}\right) \in \mathbb{Z}^{k} \backslash\{\mathbf{0}\}$ and $p \in \mathbb{Z}$; and

$$
\max _{1 \leq i \leq k}\left|q \varphi_{i}(s)-p_{i}\right| \leq \mu N^{-1} \quad \text { and } \quad|q| \leq \mu N^{k}
$$

for $q \in \mathbb{Z} \backslash\{0\}$ and $\left(p_{1}, \ldots, p_{k}\right) \in \mathbb{Z}^{k}$.

1.1. Sketch of the proof of Theorem 1.3. Let $I=[a, b]$. We will treat $G$ as a subset of $L$ via the homomorphism $\rho$. We consider the normalized parameter measure, say $\nu$, on the segment $\left\{u(\varphi(s)) x_{0}: s \in\right.$ $I\}$ on $L / \Lambda$. Take any sequence $t_{i} \rightarrow \infty$. Let $a_{t_{i}} \nu$ denote the translate of $\nu$ concentrated on the curve $a_{t_{i}} u(\varphi(I)) x_{0}$. By Dani-Margulis criterion for nondivergence, if for every compact set $\mathcal{F} \subset L / \Lambda$, we have

$$
a_{t_{i}} \nu(\mathcal{F}):=\frac{1}{|b-a|}\left|\left\{s \in[a, b]: a_{t_{i}} u(\varphi(s)) x_{0} \in \mathcal{F}\right\}\right| \rightarrow 0 \quad \text { as } i \rightarrow \infty,
$$

then the following algebraic condition holds: There exists a finite dimensional representation $V$ of $G$ and a nonzero vector $v \in V$ such that

$$
\lim _{i \rightarrow \infty} a_{t_{i}} u(\varphi(s)) v \rightarrow 0, \quad \forall s \in I .
$$

The 'Basic Lemma' (Proposition 4.2), which is one of the main tools developed in this article, says that if $\{\varphi(s): s \in I\}$ affinely spans $\mathbb{R}^{n-1}$ then (1.9) cannot hold.

Therefore using the Dani-Margulis criterion we deduce that given any $\epsilon>0$ there exists a large compact set $\mathcal{F} \subset L / \Lambda$ such that $\left(a_{t_{i}} \nu\right)(\mathcal{F}) \geq$ $1-\epsilon$. As a consequence, there exists a probability measure $\mu$ on $L / \Lambda$ such that after passing to a subsequence, $a_{t_{i}} \nu \stackrel{i \rightarrow \infty}{\longrightarrow} \mu$ with respect to the weak-* topology.

At this stage, we note that $u(\varphi(I)) x_{0}$ is contained in a strongly unstable leaf for the action of $a_{t}$ on $L / \Lambda$. Then for each $s_{0} \in I$ if $\dot{\varphi}\left(s_{0}\right)$ denotes the derivative of $\varphi$ at $s_{0}$, then

$$
\varphi(s)-\varphi\left(s_{0}\right)=\left(s-s_{0}\right) \dot{\varphi}\left(s_{0}\right)+O\left(\left(s-s_{0}\right)^{2}\right) .
$$

Therefore for any large $t>0$, the translated curve

$$
\left\{a_{t} u(\varphi(s)) x_{0}=u\left(e^{n t}\left(\varphi(s)-\varphi\left(s_{0}\right)\right)\right)\left(a_{t} u\left(\varphi\left(s_{0}\right)\right) x_{0}\right):\left|s-s_{0}\right|<\delta_{t}\right\}
$$

stays very close to the unipotent trajectory

$$
\left\{u\left(e^{n t}\left(s-s_{0}\right) \dot{\varphi}\left(s_{0}\right)\right)\left(a_{t} u\left(\varphi\left(s_{0}\right)\right) x_{0}\right):\left|s-s_{0}\right|<\delta_{t}\right\},
$$

if we choose $\delta_{t}>0$ such that $e^{n t} \delta_{t} \rightarrow \infty$, but $e^{n t} \delta_{t}^{2} \rightarrow 0$ as $t \rightarrow \infty$. Note that the length of the unipotent trajectory (1.12) is about $e^{n t} \delta_{t}\left\|\dot{\varphi}\left(s_{0}\right)\right\|$ and hence it is very long. Our difficultly is that the direction $\dot{\varphi}\left(s_{0}\right)$ of the flow varies with $s_{0}$. 
In order to take care of this problem, instead of translating the original curve, we twist it by $z(s) \subset \mathrm{Z}_{\mathrm{G}}(A) \cong A \times \mathrm{SL}(n-1, \mathbb{R})$ so that $z(s) u(\dot{\varphi}(s)) z(s)^{-1}=u\left(e_{1}\right)$ for all $s \in I$; here $A=\left\{a_{t}: t \in \mathbb{R}\right\}, e_{1}$ is a fixed nonzero vector in $\mathbb{R}^{n-1}$ and we assume that $\dot{\varphi}(s) \neq 0$ for all $s \in I$. We take another curve: $\left\{z(s) u(\varphi(s)) x_{0}: s \in I\right\}$ and associate a normalized parameter measure $\nu^{\prime}$ on it. Since $z(I)$ is contained in a compact set, we conclude that after passing to a subsequence $a_{t_{i}} \nu^{\prime}$ converges to a probability measure $\nu^{\prime}$ as $i \rightarrow \infty$. Now one can show that $\nu^{\prime}$ is invariant under the flow $\left\{u\left(s e_{1}\right): s \in \mathbb{R}\right\}$. Then we will be in the situation where we can use Ratner's theorem and the so called 'linearization technique'. Using both and the 'Basic Lemma', we will show that there exist a nonzero vector $v \in V$, which is algebraically associated to $x_{0}$, and a curve $I \ni s \mapsto w(s) \in V$ such that

$$
\lim _{t \rightarrow \infty} a_{t} u(\varphi(s)) v \rightarrow w(s), \quad \forall s \in I .
$$

Again using an extension of the 'Basic lemma' (Corollary 4.6) we show that $v$ is fixed by $G$. From this very restrictive situation, we further deduce that the measure $\nu^{\prime}$ is $H$-invariant, where $H$ is the smallest closed subgroup of $L$ containing $G$ such that the orbit $H x_{0}$ is closed. Since the modification of $\nu$ to obtain $\nu^{\prime}$ was only by elements centralizing $A$ in $G$, and since we have shown that $\nu^{\prime}$ is invariant under $\mathrm{Z}_{\mathrm{G}}(A)$, we obtain that $\nu=\nu^{\prime}$, and hence $\nu$ is $H$-invariant. This will prove Theorem 1.3 .

\subsection{Variations of the equidistribution result.}

1.2.1. Expanding translates of any curve. The following variation of Theorem 1.3 is more natural.

Theorem 1.5. Let $\boldsymbol{\psi}:[a, b] \rightarrow \operatorname{SL}(n, \mathbb{R})$ be an analytic curve such that the image of the first row of this curve is not contained in a proper subspace of $\mathbb{R}^{n}$. Let the notation be as in the statement of Theorem 1.3. Then

$$
\frac{1}{b-a} \int_{a}^{b} f\left(\rho\left(a_{t} \boldsymbol{\psi}(s)\right) x_{0}\right) d s \stackrel{t \rightarrow \infty}{\longrightarrow} \int_{H x_{0}} f d \mu_{H} .
$$

The above result will be deduced from Theorem 1.2 by using [17, Lemma 5.1].

1.2.2. Uniform versions. First we state the basic uniform version of Theorem 1.2 ,

Theorem 1.6. Let the notation be as in Theorem 1.2. Then given any compact set $\mathcal{K} \subset G / \Gamma$, a bounded continuous function $f$ on $G / \Gamma$ and 
an $\epsilon>0$, there exists $t_{0}>0$ such that for any $x \in \mathcal{K}$ and $t \geq t_{0}$, we have

$$
\begin{gathered}
\left|\int_{a}^{b} f\left(a_{t} u(\varphi(s)) x\right) d s-\int f d \mu_{G}\right| \leq|b-a| \epsilon \\
\left|\int_{a}^{b} f\left(a_{t}^{\prime} u^{\prime}(\varphi(s)) x\right) d s-\int f d \mu_{G}\right| \leq|b-a| \epsilon .
\end{gathered}
$$

The following result is a general uniform version for Theorem 1.3.

Theorem 1.7. Let $\varphi:[a, b] \rightarrow \mathbb{R}^{n-1}$ be an analytic curve whose image is not contained in a proper affine subspace of $\mathbb{R}^{n-1}$. Let $L$ be a Lie group, $\Lambda$ a lattice in $L$ and $\pi: L \rightarrow L / \Lambda$ the quotient map. Let $\rho: G \rightarrow L$ be a continuous homomorphism. Let $\mathcal{K}$ be a compact subset of $L / \Lambda$. Then given $\epsilon>0$ and a bounded continuous function $f$ on $L / \Lambda$, there exist finitely many proper closed subgroups $H_{1}, \ldots, H_{r}$ of $L$ such that for each $1 \leq i \leq r, H_{i} \cap \Lambda$ is a lattice in $H_{i}$ and there exists a compact set

$$
C_{i} \subset N\left(H_{i}, \rho(G)\right):=\left\{g \in L: \rho(G) g \subset g H_{i}\right\}
$$

such that the following holds: Given any compact set

$$
F \subset \mathcal{K} \backslash \cup_{i=1}^{r} \pi\left(C_{i}\right)
$$

there exists $t_{0}>0$ such that for any $x \in F$ and any $t \geq t_{0}$..

$$
\left|\frac{1}{b-a} \int_{a}^{b} f\left(\rho\left(a_{t} u(\varphi(s))\right) x\right) d s-\int_{L / \Lambda} f d \mu_{L}\right|<\epsilon .
$$

If $L$ is an algebraic group then the sets $N\left(H_{i}, \rho(G)\right)$ are algebraic subvarieties of $L$. Therefore unless $H$ contains a normal subgroup of $L$ containing $G$, the set $\cup_{i=1}^{r} \pi\left(C_{i}\right)$ is contained in a finite union of relatively compact lower dimensional submanifolds of $L / \Lambda$.

From the above result the following statement for translates of any curve can be deduced using [17, Lemma 5.1].

Theorem 1.8. Let $\boldsymbol{\psi}:[a, b] \rightarrow \operatorname{SL}(n, \mathbb{R})$ be an analytic curve such that the image of the first row of this curve is not contained in a proper subspace of $\mathbb{R}^{n}$. Let the notation be as in the statement of Theorem 1.7 . Then given any compact set $F \subset \mathcal{K} \backslash \cup_{i=1}^{r} \pi\left(C_{i}\right)$ there exists $t_{0}>0$ such that for any $x \in F$ and $t \geq t_{0}$, we have

$$
\left|\frac{1}{b-a} \int_{a}^{b} f\left(\rho\left(a_{t} \boldsymbol{\psi}(s)\right) x\right) d s-\int_{L / \Lambda} f d \mu_{L}\right|<\epsilon .
$$


In the next section we will deduce the number theoretic consequences from the equidistribution statement. Rest of the article closely follows the strategy laid out in $\$ 1.1$. The basic lemma and its consequences are proved in $\$ 4$.

Acknowledgement. I am very greatful to Shahar Mozes and Elon Lindenstrauss for the extensive discussions which have significantly contributed to the process of obtaining the 'Basic Lemma'. Thanks are due to D. Kleinbock, B. Weiss, Y. Bugeaud and W. Schmidt for their remarks on number theoretic implications of the results considered here. I would like to thank my wife for her encourgement.

\section{Deduction of Theorem 1.4 from Theorem 1.3}

The argument given below is based on [11, §2.1]. Let the homomorphism $\rho(g)=(g, \sigma(g))$ from $G$ to $L=G \times G$, and other notation be as in $\$ 1.0 .2$. Let $n=k+1$. Let $\left(q_{1}, \ldots, q_{k}\right) \in \mathbb{Z}^{k}, p \in \mathbb{Z}, q \in \mathbb{Z}$ and $\left(p_{1}, \ldots, p_{k}\right) \in \mathbb{Z}^{k}$. Let $N \in \mathbb{N}$ and put $t=\log (N)$. We consider the standard action of $G \times G$ on $\mathbb{R}^{n} \times \mathbb{R}^{n}$. For $s \in[a, b]$, we have

$$
\begin{aligned}
(\boldsymbol{\zeta}(N, s), \boldsymbol{\eta}(N, s)) & :=\rho\left(a_{t} u(\varphi(s))\right)\left(\left[\begin{array}{c}
p \\
q_{1} \\
\vdots \\
q_{k}
\end{array}\right],\left[\begin{array}{c}
p_{k} \\
\vdots \\
p_{1} \\
q
\end{array}\right]\right) \\
& =\left(\left[\begin{array}{c}
N^{k}\left(p+\sum_{i=1}^{k} q_{i} \varphi_{i}(s)\right) \\
N^{-1} q_{1} \\
\vdots \\
N^{-1} q_{k}
\end{array}\right],\left[\begin{array}{c}
N\left(q \varphi_{1}(s)+p_{k}\right) \\
\vdots \\
N\left(q \varphi_{k}(s)+p_{1}\right) \\
N^{-k} q
\end{array}\right]\right) .
\end{aligned}
$$

We now fix $0<\mu<1$. Let

$$
B_{\mu}=\left\{\left(\xi_{1}, \ldots, \xi_{n}\right) \in \mathbb{R}^{n}: \max _{1 \leq i \leq n}\left|\xi_{i}\right| \leq \mu\right\} .
$$

Then (1.6) is equivalent to $\boldsymbol{\zeta}(N, s) \in B_{\mu}$ and (1.7) is equivalent to $\boldsymbol{\eta}(N, s) \in B_{\mu}$.

Let $\Omega$ denote the space of unimodular lattices in $\mathbb{R}^{n}$. Note that $G$ acts transitively on $\Omega$ and the stabilizer of $\mathbb{Z}^{n}$ is $\Gamma$. Similarly $L$ acts transitively on $\Omega \times \Omega$ and the stabilizer of $x_{0}:=\left(\mathbb{Z}^{n}, \mathbb{Z}^{n}\right)$ is $\Lambda$. Thus $G / \Gamma \cong \Omega$ and $L / \Lambda \cong \Omega \times \Omega$.

Let

$$
K_{\mu}=\left\{\Delta \in \Omega: \Delta \cap B_{\mu}=\{\mathbf{0}\}\right\} .
$$

As we observed above for any $s \in[a, b]$ and $N \in \mathbb{N}$, the inequalities (1.6) and (1.7) are simultaneously insoluble, if for $t=\log N$,

$$
\rho\left(a_{t} u(\varphi(s))\right) x_{0} \in K_{\mu} \times K_{\mu} .
$$


As noted earlier there exists a $\rho(G)$-invariant probability measure on $\rho(G) x_{0} \subset \Omega \times \Omega$, say $\lambda$. Since $\mu<1, K_{\mu} \times K_{\mu}$ contains an open neighbourhood of $x_{0}$. Hence there exists $\epsilon>0$ such that

$$
\lambda\left(K_{\mu} \times K_{\mu}\right)>\epsilon .
$$

Therefore there exists a continuous function $0 \leq f \leq 1$ such that

$$
\operatorname{supp}(f) \subset K_{\mu} \times K_{\mu} \text { and } \int f d \lambda>\epsilon / 2
$$

Let $J$ be any subinterval of $[a, b]$ with nonempty interior. Then by Theorem 1.3, there exists $N_{0}>0$, such that for all $N \geq N_{0}$, and $t=\log N$,

$$
\frac{1}{|J|} \int_{s \in J} f\left(\rho\left(a_{t} u(\varphi(s))\right) x_{0}\right) d s \geq \epsilon / 4
$$

where $|\cdot|$ denotes the Lebesgue measure on $\mathbb{R}$.

Let $\mathcal{N} \subset \mathcal{N}$ be an infinite set. Let

$$
E=\left\{s \in[a, b]: \rho\left(a_{(\log N)} u(\varphi(s))\right) x_{0} \notin K_{\mu} \times K_{\mu} \text { for all large } N \in \mathcal{N}\right\} \text {. }
$$

By (2.8), for any subinterval $J \subset[a, b]$, we have $|J \cap E| \leq(1-\epsilon / 4)|J|$. Therefore $|E|=0$. In view of the observation associated to (2.5), this proves Theorem 1.4.

The Theorem 1.1 is a special case of Theorem 1.4. On the other hand, the proof of Theorem 1.1 can also be deduced directly from Theorem 1.2 in a similar way.

\section{Non-Divergence OF THE LIMITING Distribution}

Let $\varphi: I=[a, b] \rightarrow \mathbb{R}^{n-1}$ be a nonconstant analytic map. Let $x_{0} \in$ $L / \Lambda$. Given a nontrivial continuous homomorphism $\rho: G \rightarrow L$, for the sake of simplicity of notation in our study, without loss of generality, we will identify $g \in G$ with $\rho(g) \in L$. Therefore now onwards we will treat $G$ as a subgroup of $L$, where $\rho$ being an inclusion. Let $t_{i} \rightarrow \infty$ be any sequence in $\mathbb{R}$. Let $x_{i} \rightarrow x_{0}$ be a convergent sequence in $L / \Lambda$. For any $i \in \mathbb{N}$ let $\mu_{i}$ be the measure on $L / \Lambda$ defined by

$$
\int_{L / \Lambda} f d \mu_{i}:=\frac{1}{|I|} \int_{I} f\left(a_{t_{i}} u(\varphi(s)) x_{i}\right) d s, \quad \forall f \in \mathrm{C}_{\mathrm{c}}(L / \Lambda) .
$$

Theorem 3.1. Given $\epsilon>0$ there exists a compact set $\mathcal{F} \subset L / \Lambda$ such that $\mu_{i}(\mathcal{F}) \geq 1-\epsilon$ for all large $i \in \mathbb{N}$.

It may be noted that in the case of $L=G, \rho$ the identity map and $\Lambda=\mathrm{SL}(n, \mathbb{Z})$, the above result was already proved by Kleinbock and 
Margulis [10]. The rest of this section is devoted to obtaining the same conclusion in the case of arbitrary $L$ and $\Lambda$.

3.1. Let $\mathscr{H}$ denote the collection of analytic subgroups $H$ of $G$ such that $H \cap \Lambda$ is a lattice in $H$, and a unipotent one-parameter subgroup of $H$ acts ergodically with respect to the $H$-invariant probability measure on $H / H \cap \Lambda$. Then $\mathscr{H}$ is a countable collection [15, 13].

Let $\mathfrak{l}$ denote the Lie algebra associated to $L$. Let $V=\oplus_{d=1}^{\operatorname{dim} \mathfrak{l}} \wedge^{d} \mathfrak{l}$ and consider the $\left(\oplus_{d=1}^{\operatorname{dim} \mathfrak{l}} \wedge^{d} \mathrm{Ad}\right)$-action of $L$ on $V$. Given $H \in \mathscr{H}$, let $\mathfrak{h}$ denote its Lie algebra, and fix $p_{H} \in \wedge^{\operatorname{dim} \mathfrak{h}} \mathfrak{h} \backslash\{0\} \subset V$. Then

$$
\operatorname{Stab}_{L}\left(p_{H}\right)=\mathrm{N}_{\mathrm{L}}^{1}(H):=\left\{g \in \mathrm{N}_{\mathrm{L}}(H): \operatorname{det}\left(\left.(\operatorname{Ad} g)\right|_{\mathfrak{h}}\right)=1\right\} .
$$

Proposition $3.2([5])$. The orbit $\Lambda \cdot p_{H}$ is a discrete subset of $V$.

We may note that when $L$ is a real algebraic group defined over $\mathbb{Q}$ and $\Lambda=L(\mathbb{Z})$ then the above countability result and the discreteness of the orbit are straightforward to prove.

3.2. Functions with growth factor $C$ and growth order $\alpha$. Let $\mathscr{F}$ denote the $\mathbb{R}$-span of all the the coordinate functions of the map $\Upsilon: I \rightarrow \operatorname{End}(V)$ given by $\Upsilon(s)=\left(\oplus_{d=1}^{\operatorname{dim} \mathfrak{l}} \wedge^{d} \operatorname{Ad}\right)(u(\varphi(s)))$ for all $s \in \mathbb{R}$. As explained in [17, §2.1], due to [10, Proposition 3.4] the family $\mathscr{F}$ has the following growth property for some $C>0$ and $\alpha>0$ : for any subinterval $J \subset I, \xi \in \mathscr{F}$ and $r>0$,

$$
|\{s \in J:|\xi(s)|<r\}|<C\left(\frac{r}{\sup _{s \in J}|\xi(s)|}\right)^{\alpha}|J| .
$$

As a direct consequence of this condition, we have the following [10]: Fix any norm $\|\cdot\|$ on $V$.

Proposition 3.3. Given any $\epsilon>0$ and $r>0$, there exists $R>0$ such that for any $h_{1}, h_{2} \in L$ and a subinterval $J \subset I$, one of the following holds:

I) $\sup _{t \in J}\left\|h_{1} u(\varphi(t)) h_{2} p_{H}\right\|<R$.

II)

$$
\begin{aligned}
\mid\left\{t \in J:\left\|h_{1} u(\varphi(t)) h_{2} p_{H}\right\|\right. & \leq r\} \mid \\
& \leq \epsilon\left|\left\{t \in J:\left\|h_{1} u(\varphi(t)) h_{2} p_{H}\right\| \leq R\right\}\right| .
\end{aligned}
$$




\subsection{The non-diverergence criterion.}

Proposition 3.4. There exist closed subgroups $W_{1}, \ldots, W_{r} \in \mathscr{H}$ (depending only on $L$ and $\Lambda$ ) such that the following holds: Given $\epsilon>0$ and $R>0$, there exists a compact set $\mathcal{F} \subset L / \Lambda$ such that for any $h_{1}, h_{2} \in L$ and a subinterval $J \subset I$, one of the following conditions is satisfied:

I) There exists $\gamma \in \Lambda$ and $i \in\{1, \ldots, r\}$ such that

$$
\sup _{s \in J}\left\|h_{1} u(\varphi(s)) h_{2} p_{H}\right\|<R .
$$

II) $\frac{1}{|J|}\left|\left\{s \in J:\left(h_{1} u(\varphi(s)) h_{2}\right) \Lambda / \Lambda \in \mathcal{F}\right\}\right| \geq 1-\epsilon$.

Proof. The result follows from the argument as in [16, Theorem 2.2] using the earlier results of Dani and Margulis [4]; as well as its extensions due to Kleinbock and Margulis [10]. The main difference is that instead of growth properties of polynomial functions, one uses the similar properties of functions in $\mathscr{F}$ as given by Proposition 3.3.

Proof of Theorem 3.1. Take any $\epsilon>0$. Take a sequence $R_{k} \rightarrow 0$ as $k \rightarrow \infty$. For each $k \in \mathbb{N}$, let $\mathcal{F}_{k} \subset L / \Lambda$ be a compact set as determined by Proposition 3.4 for these $\epsilon$ and $R_{k}$. If the theorem fails to hold, then for each $k \in \mathbb{N}$ we have $\mu_{i}\left(\mathcal{F}_{k}\right)<1-\epsilon$ for all large $i$. Therefore after passing to a subsequences of $\left\{\mu_{i}\right\}$, we may assume that $\mu_{i}\left(\mathcal{F}_{i}\right)<1-\epsilon$ for all $i$. Then by Proposition [3.4, after passing to a subsequence, we may assume that there exists $W \in \mathscr{H}$ such that for each $i$ there exists $\gamma_{i} \in \Lambda$ such that

$$
\left\|\sup _{s \in I} a_{t_{i}} u(\varphi(s)) \gamma_{i} p_{W}\right\| \leq R_{i} \stackrel{i \rightarrow \infty}{\longrightarrow} 0 .
$$

By Proposition [3.2, there exists $r_{0}>0$ such that $\left\|\gamma_{i} p_{W}\right\| \geq r_{0}$ for each $i$. We put $v_{i}=\gamma_{i} p_{W} /\left\|\gamma_{i} p_{W}\right\|$. Then $v_{i} \rightarrow v \in V$ and $\|v\|=1$. Therefore

$$
\sup _{s \in I}\left\|a_{t_{i}} u(\varphi(s)) v_{i}\right\| \leq R_{i} / r_{0} \rightarrow 0 \quad \text { as } i \rightarrow \infty .
$$

Define

$$
\begin{aligned}
& V^{-}=\left\{w \in V: \lim _{t \rightarrow \infty} a_{t} w=0\right\} \\
& V^{+}=\left\{w \in V: \lim _{t \rightarrow \infty}\left(a_{t}\right)^{-1} w=0\right\} \\
& V^{0}=\left\{w \in V: a_{t} w=w, \quad \forall t \in \mathbb{R}\right\} .
\end{aligned}
$$

Since $\left\{a_{t}\right\}$ acts on $V$ via commuting $\mathbb{R}$-diagonalizable matrices, we have that $V=V^{+} \oplus V^{0} \oplus V^{-}$. Let $\pi_{0}: V \rightarrow V^{0}$ denote the associated 
projection. Then from (3.5) we conclude that

$$
u(\varphi(s)) v \subset V^{-}, \quad \forall s \in I .
$$

The 'Basic Lemma' (Proposition 4.2) proved in the next section states that for any finite dimensional linear representation $V$ of $G$, any $v \in V \backslash\{0\}$ and any $\mathcal{B} \subset \mathbb{R}^{n-1}$ which is not contained in a proper affine subspace, if

$$
u(\varphi(e)) v \in V^{-}+V^{0}, \quad \forall e \in \mathcal{B}
$$

then

$$
\pi_{0}(u(\varphi(e)) v) \neq 0, \quad \forall e \in \mathcal{B} .
$$

By our hypothesis (3.9) implies (3.10) but contradicts its consequence (3.11).

As a consequence of Theorem 3.1 we deduce the following:

Corollary 3.5. After passing to a subsequence, $\mu_{i} \rightarrow \mu$ in the space of probability measures on $L / \Lambda$ with respect to the weak-* topology.

Before we proceed further from here, we will give a proof of the Basic lemma and obtain its consequence, which will be used in the later sections.

\section{Dynamics of Linear actions of intertwined $\operatorname{SL}(2, \mathbb{R})$ 'S}

A triple $(X, H, Y)$ of elements of a Lie algebra is called an $\mathfrak{s l}_{2}$-triple if

$$
[X, Y]=H, \quad[H, X]=2 X, \quad[H, Y]=-2 Y .
$$

The following observation on linear dynamics of $\mathrm{SL}_{2}$-action played a crucial role in understanding limiting distributions of expanding translates of curves under the geodesic flows on hyperbolic manifolds [17].

Lemma 4.1 ([17, Lemma 2.3]). Let $W$ be a finite dimensional irreducible representation of an $\mathfrak{s l}_{2}$-triple $(X, H, Y)$. Let $W^{-}$(respectively, $W^{+}$) denote the sum of strictly negative (respectively, strictly positive) eigenspaces of $H$. Let $\pi_{+}: W \rightarrow W^{+}$denote the projection parallel to the eigenspaces of $H$. Then

$$
v \in W^{-} \backslash\{0\} \Longrightarrow \pi_{+}(\exp (X) v) \neq 0 .
$$

The main goal of this section is to obtain a similar result on linear dynamics of $\mathrm{SL}(n, \mathbb{R})$-actions by considering intertwined actions of copies of $\operatorname{SL}(2, \mathbb{R})$ 's contained in $\operatorname{SL}(n, \mathbb{R})$. 
4.0.1. Notation. Let $\mathcal{A}=\operatorname{diag}((n-1),-1, \ldots,-1) \in \mathfrak{s l}(n, \mathbb{R})$. Then $a_{t}=\exp (t \mathcal{A})$ for all $t \in \mathbb{R}$. Define $A=\left\{a_{t}: t \in \mathbb{R}\right\}$ and $\mathfrak{a}=\operatorname{Lie}(A)=$ $\mathbb{R} \cdot \mathcal{A}$. Consider a linear representation of $G$ on a finite dimensional vector space $V$. For $\mu \in \mathbb{R}$, define

$$
V^{\mu}=\{v \in V: \mathcal{A} v=\mu v\} .
$$

Let $\pi_{\mu}: V \rightarrow V^{\mu}$ denote the projection parallel to the eigen spaces of A. Put

$$
\begin{aligned}
& V^{-}=\sum_{\mu<0} V^{\mu}, \quad V^{+}=\sum_{\mu>0} V^{\mu} \\
& \pi_{-}=\sum_{\mu<0} \pi_{\mu}, \quad \pi_{+}=\sum_{\mu>0} \pi_{\mu} .
\end{aligned}
$$

An affine basis of $\mathbb{R}^{n-1}$ is a set $\mathcal{B} \subset \mathbb{R}^{n-1}$ such that for any $e \in \mathcal{B}$, the set $\left\{e^{\prime}-e: e^{\prime} \in \mathcal{B} \backslash\{e\}\right\}$ is a basis of $\mathbb{R}^{n-1}$.

Proposition 4.2 (Basic Lemma). Let the notation be as above. Given an affine basis $\mathcal{B}$ of $\mathbb{R}^{n-1}$ and a nonzero vector $v \in V$, suppose that

$$
u(e) v \in V^{0}+V^{-}, \quad \forall e \in \mathcal{B} .
$$

Then

$$
\pi_{0}(u(e) v) \neq 0, \quad \forall e \in \mathcal{B} .
$$

Proof. By (4.6) there exists $\mu_{0} \leq 0$ and $e_{0} \in \mathcal{B}$ such

$$
\begin{aligned}
\pi_{\mu_{0}}\left(u\left(e_{0}\right) v\right) \neq 0, & \text { and } \\
\pi_{\mu}(u(e) v)=0, & \forall \mu>\mu_{0} \text { and } \forall e \in \mathcal{B} .
\end{aligned}
$$

We write the basis $\left\{e-e_{0}: e \in \mathcal{B} \backslash\left\{e_{0}\right\}\right\} \mathbb{R}^{n-1}$ as $\left\{e_{1}, \ldots, e_{n-1}\right\}$. Put $v_{0}=u\left(e_{0}\right) v$. Then

$$
\begin{aligned}
\pi_{\mu_{0}}\left(v_{0}\right) \neq 0 & \text { and } \\
\pi_{\mu}\left(u\left(e_{i}\right) v_{0}\right)=0 & \text { for all } \mu>\mu_{0} \text { and } 1 \leq i \leq n-1 .
\end{aligned}
$$

To prove (4.7) we need to show that

$$
\begin{aligned}
\mu_{0}=0 & \text { and } \\
\pi_{0}\left(u\left(e_{i}\right) v_{0}\right) & \neq 0, \quad \forall 1 \leq i \leq n-1 .
\end{aligned}
$$

Let the set $\left\{f_{1}, \ldots, f_{n-1}\right\}$ consisting of real $((n-1) \times 1)$ column matrices be the dual to the basis $\left\{e_{1}, \ldots, e_{n-1}\right\}$ of $\mathbb{R}^{n-1}$ consisting of $(1 \times(n-1))$-row matrices; that is,

$$
e_{i} f_{j}=\delta_{i, j}, \quad \forall i, j \in\{1, \ldots, n-1\},
$$

For $i \in\{1, \ldots, n-1\}$, let

$$
X_{i}:=X\left(e_{i}\right)=\left[\begin{array}{cc}
0 & e_{i} \\
& \mathbf{0}_{n-1}
\end{array}\right] \quad \text { and } \quad Y_{i}:=Y\left(f_{i}\right)=\left[\begin{array}{cc}
0 & \\
f_{i} & \mathbf{o}_{n-1}
\end{array}\right],
$$


where $\mathbf{0}_{n-1}$ is the $((n-1) \times(n-1))$-zero matrix. Then $u\left(e_{i}\right)=\exp \left(X_{i}\right)$. Let

$$
H_{i}:=\left[X_{i}, Y_{i}\right]=\left[\begin{array}{ll}
e_{i} f_{i} & \\
& -f_{i} e_{i}
\end{array}\right]=\left[\begin{array}{ll}
1 & \\
& -f_{i} e_{i}
\end{array}\right] \in \mathfrak{s l}(n, \mathbb{R}) .
$$

Then $\left(X_{i}, H_{i}, Y_{i}\right)$ is an $\mathfrak{s l}_{2}$-triple. Let $\mathfrak{g}_{i}=\operatorname{span}\left\{X_{i}, H_{i}, Y_{i}\right\} \subset \mathfrak{s l}(n, \mathbb{R})$. By (4.14)

$$
\sum_{i=1}^{n-1} f_{i} e_{i}=I_{n-1},
$$

the $((n-1) \times(n-1))$-identity matrix. Therefore

$$
\mathcal{A}=H_{1}+\cdots+H_{n-1} \text {. }
$$

Also

$$
\mathfrak{b}=\operatorname{span}\left\{H_{i}: i=1, \ldots, n-1\right\}
$$

is a maximal $\mathbb{R}$-diagonalizable subalgebra of $\mathfrak{s l}(n, \mathbb{R})$. Moreover

$$
\left[H, \mathfrak{g}_{\mathfrak{i}}\right]=\mathfrak{g}_{\mathfrak{i}}, \quad \forall H \in \mathfrak{b} .
$$

Thus $\mathfrak{b}+\mathfrak{g}_{\mathfrak{i}}$ is a reductive Lie algebra which is isomorphic to $\mathbb{R}^{n-2} \oplus \mathfrak{s l}_{2}$. Note that the Lie groups associated to these $\mathfrak{g}_{i}$ 's are our intertwined copies of $\mathrm{SL}_{2}$ 's, and we want to study their joint linear dynamics.

For a linear functional $\delta \in \mathfrak{b}^{*}$, let

$$
V(\delta)=\{v \in V: H v=\delta(H) v\} .
$$

The set $\Delta=\left\{\delta \in \mathfrak{b}^{*}: V(\delta) \neq 0\right\}$ is finite and $V=\oplus_{\delta \in \Delta} V(\delta)$. Let $q_{\boldsymbol{\lambda}}: V \rightarrow V(\delta)$ be the associated projection.

Claim 4.2.1. Let $\delta \in \Delta$ be such that $v(\delta):=q_{\delta}\left(\pi_{\mu_{0}}\left(v_{0}\right)\right) \neq 0$. Then $\delta\left(H_{i}\right) \geq 0$ for all $1 \leq i \leq n-1$.

To prove the claim, take any $1 \leq i \leq n-1$. Consider the decomposition

$$
V=W_{1} \oplus \cdots \oplus W_{s}
$$

where $W_{j}$ 's are irreducible subspaces for the action of the Lie subalgebra $\mathfrak{b}+\mathfrak{g}_{i}$ and $s \in \mathbb{N}$. Therefore each $W_{j}$ is an irreducible representation of the $\mathfrak{s l}_{2}$-triple $\left(X_{i}, H_{i}, Y_{i}\right)$. Let $P_{j}: V \rightarrow W_{j}$ denote the associated projection. We note that

$$
\begin{gathered}
\pi_{\mu} \circ P_{j}=P_{j} \circ \pi_{\mu}, \quad \text { for all } 1 \leq j \leq s \text { and } \mu \in \mathbb{R}, \text { and } \\
q_{\delta} \circ P_{j}=P_{j} \circ q_{\delta}, \quad \text { for all } 1 \leq j \leq s .
\end{gathered}
$$

There exists $1 \leq j \leq s$ such that

$$
P_{j}(v(\delta)) \neq 0,
$$


we take any such $j$. In particular, by (4.23) and (4.24),

$$
W_{j} \cap V_{\mu_{0}} \ni P_{j}\left(\pi_{\mu_{0}}\left(v_{0}\right)\right) \neq 0 .
$$

By the standard description of finite dimensional representations of $\mathfrak{s l}_{2}$, let $k \geq 0$ and $w_{-k} \in W_{j}$ be such that

$$
Y_{i} \cdot w_{-k}=0 \text { and } H_{i} \cdot w_{-k}=-k \cdot w_{-k} .
$$

For any $r \geq 0$, put $w_{-k+2 r}:=X_{i}^{r} \cdot w_{-k}$. Then

$$
H_{i} \cdot w_{-k+2 r}=(-k+2 r) w_{-k+2 r}
$$

and $W_{j}=\operatorname{span}\left\{w_{-k}, \ldots, w_{k}\right\}$. Since $\left[H_{i}, \mathfrak{b}\right]=0$ and $W_{j}$ is $\mathfrak{b}$-invariant, for each $0 \leq r \leq k$, there exists $\delta_{r} \in \Delta$ such that $w_{-k+2 r} \in V\left(\delta_{r}\right)$ and

$$
\delta_{r} \neq \delta_{r^{\prime}}, \quad \text { if } r \neq r^{\prime} .
$$

Put $\lambda=\delta_{0}(\mathcal{A})$. Then

$$
\mathcal{A} \cdot w_{-k}=\lambda \cdot w_{-k}
$$

Since $\left[\mathcal{A}, X_{i}\right]=n$, we have

$$
\mathcal{A} \cdot w_{-k+2 r}=(\lambda+n r) w_{-k+2 r}, \quad \forall 0 \leq r \leq k .
$$

Thus, if $P_{j}\left(V_{\mu}\right) \neq 0$ for any $\mu$, then $P_{j}\left(V_{\mu}\right) \subset \mathbb{R} \cdot w_{-k+2 r}$ for some $r \geq 0$ such that $\lambda+n r=\mu$.

Therefore by (4.26) there exists $r_{0} \geq 0$ such that

$$
\begin{aligned}
\mu_{0} & =\lambda+n r_{0} \quad \text { and } \\
W_{j} \cap V_{\mu_{0}} & =\mathbb{R} \cdot w_{-k+2 r_{0}} .
\end{aligned}
$$

Recall that $u\left(e_{i}\right)=\exp \left(X_{i}\right)$. By (4.11) and (4.23), for all $\mu>\mu_{0}$, we have

$$
\pi_{\mu}\left(\exp \left(X_{i}\right) P_{j}\left(v_{0}\right)\right)=\pi_{\mu}\left(P_{j}\left(\exp \left(X_{i}\right) v_{0}\right)\right)=P_{j}\left(\pi_{\mu}\left(\exp \left(X_{i}\right) v_{0}\right)\right)=0 .
$$

Therefore

$$
\left\{P_{j}\left(v_{0}\right), \exp \left(X_{i}\right) P_{j}\left(v_{0}\right)\right\} \subset \operatorname{span}\left\{w_{-k}, \ldots, w_{-k+2 r_{0}}\right\} .
$$

Therefore by Lemma 4.1 applied to the $\mathfrak{s l}_{2}$-triple $\left(X_{i}, H_{i}, Y_{i}\right)$, since $P_{j}\left(v_{0}\right) \neq 0$, we have

$$
-k+2 r_{0} \geq 0
$$

By (4.25), (4.29) and (4.33),

$$
0 \neq P_{j}(v(\delta))=P_{j}\left(q_{\delta}\left(\pi_{\mu_{0}}\left(v_{0}\right)\right)\right)=q_{\delta}\left(P_{j}\left(\pi_{\mu_{0}}\left(v_{0}\right)\right)\right)=P_{j}\left(\pi_{\mu_{0}}\left(v_{0}\right)\right) .
$$


Also

$$
H_{i}\left(P_{j}(v(\delta))\right)=P_{j}\left(H_{i}(v(\delta))\right)=P_{j}\left(\delta\left(H_{i}\right) v(\delta)\right)=\delta\left(H_{i}\right) \cdot P_{j}(v(\delta)),
$$

and

$$
H_{i}\left(P_{j}\left(\pi_{\mu_{0}}\left(v_{0}\right)\right)\right)=\left(-k+2 r_{0}\right) P_{j}\left(\pi_{\mu_{0}}\left(v_{0}\right)\right) .
$$

Therefore by (4.37)

$$
\delta\left(H_{i}\right)=-k+2 r_{0} \geq 0 .
$$

This completes the proof of the Claim 4.2.1.

Since $\pi_{\mu_{0}}\left(v_{0}\right) \neq 0$, there exists $\delta \in \Delta$ such that

$$
v(\delta):=q_{\delta}\left(\pi_{\mu_{0}}\left(v_{0}\right)\right) \neq 0 .
$$

Now since $\mathcal{A} \cdot v(\delta)=\mu_{0} v(\delta)$ and $\mathcal{A}=H_{1}+\cdots+H_{n-1}$, by Claim 4.2.1

$$
0 \geq \mu_{0}=\sum_{i=1}^{n-1} \delta\left(H_{i}\right) \geq 0 .
$$

Therefore

$$
\mu_{0}=0 \quad \text { and } \quad \delta\left(H_{i}\right)=0, \quad \forall 1 \leq i \leq n-1 .
$$

Thus (4.12) is verified.

Going back to the representation $W_{j}$ of $\mathfrak{g}_{i}$ considered above, by (4.41) and (4.43) $-k+2 r_{0}=\delta\left(H_{i}\right)=0$ and $\mathcal{A} \cdot w_{0}=0$. Therefore by (4.36), we have

$$
\left\{P_{j}\left(v_{0}\right), \exp \left(X_{i}\right) P_{j}\left(v_{0}\right)\right\} \subset \operatorname{span}\left\{w_{-k}, \ldots, w_{0}\right\} .
$$

Therefore, since $P_{j}\left(v_{0}\right) \neq 0$, by Lemma 4.1

$$
\exp \left(X_{i}\right) P_{j}\left(v_{0}\right) \not \subset \operatorname{span}\left\{w_{-k}, \ldots, w_{-2}\right\} .
$$

Hence by (4.33) and (4.23),

$$
P_{j}\left(\pi_{0}\left(\exp \left(X_{i}\right) v_{0}\right)\right)=\pi_{0}\left(P_{j}\left(\exp \left(X_{i}\right) v_{0}\right)\right)=\pi_{0}\left(\exp \left(X_{i}\right) P_{j}\left(v_{0}\right)\right) \neq 0 .
$$

Therefore $\pi_{0}\left(\exp \left(X_{i}\right) v_{0}\right) \neq 0$. Thus (4.13) is verified.

Consider the linear action of $\mathrm{Z}_{\mathrm{G}}(A)$ on $\mathbb{R}^{n-1}$ such that

$$
u(g \cdot e)=g u(e) g^{-1}, \forall g \in \mathrm{Z}_{\mathrm{G}}(A), \forall e \in \mathbb{R}^{n-1} .
$$

Note that under this action $\mathrm{Z}_{\mathrm{G}}(A)$ maps onto $\mathrm{GL}(n-1, \mathbb{R})$. We also note that for any basis $\mathcal{C}$ of $\mathbb{R}^{n-1}$, the set

$$
D_{\mathcal{C}}:=\left\{g \in \mathrm{Z}_{\mathrm{G}}(A) \text { : each } e \in \mathcal{C} \text { is an eigenvector of } g\right\}
$$

is a maximal $\mathbb{R}$-diagonalizable subgroup of $G$. 
Corollary 4.3. Let the notation be as in Proposition 4.2. For any $e \in \mathcal{B}$,

$$
g \pi_{0}(u(e) v)=\pi_{0}(u(e) v), \quad \forall g \in D_{\mathcal{C}}
$$

where $\mathcal{C}=\left\{e^{\prime}-e: e^{\prime} \in \mathcal{B} \backslash\{e\}\right\}$.

Proof. As a consequence of Proposition 4.2, $\mu_{0}=0$ and $\pi_{\mu_{0}}(u(e) v) \neq 0$. Let the notation be as in the proof of Proposition 4.2. Then $u(e) v=v_{0}$. Now $\mathcal{C}=\left\{e_{1}, \ldots, e_{n-1}\right\}$ and for any $i$, we have $H_{i} e_{i}=2$ and $H_{i} e_{j}=e_{j}$ if $j \neq i$. Therefore by (4.19), $D_{\mathcal{C}}=\exp (\mathfrak{b})$.

For any $\delta \in \Delta$, if $q_{\delta}\left(\pi_{\mu_{0}}\left(v_{0}\right)\right) \neq 0$ then by (4.43)

$$
H_{i} q_{\delta}\left(\pi_{\mu_{0}}\left(v_{0}\right)\right)=\delta\left(H_{i}\right) q_{\delta}\left(\pi_{\mu_{0}}\left(v_{0}\right)\right)=0, \quad \forall 1 \leq i \leq n-1 .
$$

Therefore $H_{i} \pi_{\mu_{0}}\left(v_{0}\right)=\sum_{\delta \in \Delta} H_{i} q_{\delta}\left(\pi_{\mu_{0}}\left(v_{0}\right)\right)=0$ for all $i$. Hence $\mathfrak{b}$. $\pi_{0}\left(v_{0}\right)=0$. Therefore $D_{\mathcal{C}} \pi_{0}\left(v_{0}\right)=\pi_{0}\left(v_{0}\right)=p_{0}(u(e) v)$ and we obtain (4.49).

Corollary 4.4. Let a set $\mathcal{E} \subset \mathbb{R}^{n-1}$ and $e \in \mathcal{E}$ be such that the set $\mathcal{E}_{e}:=\left\{e^{\prime}-e: e^{\prime} \in \mathcal{E}\right\}$ is not contained a union of $n-1$ proper subspaces of $\mathbb{R}^{n-1}$. Suppose that $v \in \mathbb{R}^{n-1}$ is such that

$$
u\left(e^{\prime}\right) v \in V^{0}+V^{-}, \quad \forall e^{\prime} \in \mathcal{E} .
$$

Then

$$
\begin{gathered}
\pi_{0}(u(e) v) \neq 0 \quad \text { and } \\
\mathrm{Z}_{\mathrm{G}}(A) \subset \operatorname{Stab}_{G}\left(\pi_{0}(u(e) v)\right) .
\end{gathered}
$$

Proof. First (4.52) follows from Proposition 4.2.

Replacing $v$ by $u(e) v$ and $\mathcal{E}$ by $\mathcal{E}_{e}$, without loss of generality we may assume that $e=0 \in \mathcal{E}$ and we only need to prove that

$$
\mathrm{Z}_{\mathrm{G}}(A) \subset \operatorname{Stab}_{G}\left(\pi_{0}(v)\right)
$$

By our hypothesis there exist $\left\{b_{1}, \ldots, b_{n-1}\right\} \subset \mathcal{E}$ which is a basis of $\mathbb{R}^{n-1}$. Let $\left\{e_{i}: i=1, \ldots, n-1\right\}$ denote the standard basis of $\mathbb{R}^{n-1}$. We put $e_{0}=0$. Then there exists $z \in \mathrm{Z}_{\mathrm{G}}(A)$ such that $z b_{i}=e_{i}$ for $1 \leq i \leq n-1$. Now by (4.51),

$$
z(u(b) v)=\left(z u(b) z^{-1}\right)(z v)=u(z \cdot b)(z v) \subset V^{0}+V^{-}, \quad \forall b \in \mathcal{E} .
$$

Also $\pi_{0}(z w)=z \pi_{0}(w)$ for all $w \in V$. Therefore to prove the result, without loss of generality we can replace $\mathcal{E}$ with $z \cdot \mathcal{E}$ and $v$ with $z v$, and assume that

$$
\mathcal{B}:=\left\{e_{i}: 0 \leq i \leq n-1\right\} \subset \mathcal{E}
$$


Let $\mathcal{C}=\left\{e_{1}, \ldots, e_{n-1}\right\}$. Let $D$ denote the maximal diagonal subgroup of $\mathrm{SL}(n, \mathbb{R})$. Then $D_{\mathcal{C}}=D$ and by Corollary 4.3 ,

$$
D \subset \operatorname{Stab}\left(\pi_{0}(v)\right) \text {. }
$$

By our hypothesis, $\mathcal{E} \backslash\left\{e_{1}, \ldots, e_{n-1}\right\}$ is not contained in a proper subspace of $\mathbb{R}^{n-1}$. Therefore there exists $e_{1}^{\prime} \in \mathcal{E}$ such that

$$
e_{1}^{\prime}=\sum_{i=1}^{n-1} \lambda_{i} e_{i} \quad \text { and } \quad \lambda_{i} \neq 0, \quad \forall 1 \leq i \leq n-1 .
$$

For $\boldsymbol{x}=\left(x_{1}, \ldots, x_{n-2}\right) \in \mathbb{R}^{n-2}$, let

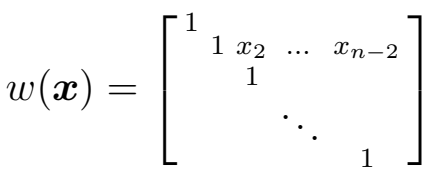

Put $\boldsymbol{x}=\left(-\lambda_{1}^{-1} \lambda_{2}, \ldots,-\lambda_{1}^{-1} \lambda_{n-1}\right)$. Then $w(\boldsymbol{x}) e_{1}=\lambda_{1}^{-1} e_{1}^{\prime}$ and $w(\boldsymbol{x}) e_{i}=$ $e_{i}$ for $i=2, \ldots, n-1$. Let $\mathcal{C}^{\prime}=\left\{e_{1}^{\prime}, e_{2}, \ldots, e_{n-1}\right\}$. Then

$$
D_{\mathcal{C}^{\prime}}=w(\boldsymbol{x})^{-1} D w(\boldsymbol{x}) \text {. }
$$

Since $\mathcal{B}^{\prime}:=\left\{0, e_{1}^{\prime}, e_{2}, \ldots, e_{n-1}\right\} \subset \mathcal{E}$, by Corollary 4.3 applied to $\mathcal{B}^{\prime}$ in place of $\mathcal{B}$, we obtain that

$$
w(\boldsymbol{x})^{-1} D w(\boldsymbol{x})=D_{\mathcal{C}^{\prime}} \subset \operatorname{Stab}\left(\pi_{0}(v)\right) .
$$

Since each coordinate of $\boldsymbol{x}$ is nonzero, the group generated by $D$ and $w(\boldsymbol{x})^{-1} D w(\boldsymbol{x})$ contains $W:=\left\{w(\boldsymbol{y}): \boldsymbol{y} \in \mathbb{R}^{n-2}\right\}$. Then by (4.57) and (4.61), $D W \subset \operatorname{Stab}_{G}\left(\pi_{0}(v)\right)$. Let $\alpha>1$. Then $W$ is the expanding horospherical subgroup of $\mathrm{Z}_{\mathrm{G}}(A)$ associated to

$$
g_{0}=\operatorname{diag}\left(1, \alpha^{n-2}, \alpha^{-1}, \ldots, \alpha^{-1}\right) \in D \text {. }
$$

Note that $W^{-}:=\left\{{ }^{t} w(v y): \boldsymbol{y} \in \mathbb{R}^{n-2}\right\}$ is the contracting horospherical subgroup of $\mathrm{Z}_{\mathrm{G}}(A)$ associated to $g_{0}$. Therefore $\pi_{0}(v)$ is stabilized by $W^{-}$. Since $\mathrm{Z}_{\mathrm{G}}(A) \cong A \cdot \mathrm{SL}(n-1, \mathbb{R})$, one verifies that $\mathrm{Z}_{\mathrm{G}}(A)$ is generated by $W, W^{-}$and $D$. Therefore $\mathrm{Z}_{\mathrm{G}}(A)$ stabilizes $\pi_{0}(v)$; that is (4.54) holds.

Lemma 4.5. Let $x_{i} \rightarrow x$ be a convergent sequence in $\mathbb{R}^{n-1}$. Suppose there exists $v \in V$ such that

$$
u\left(x_{i}\right) v \in V^{0}+V^{-}, \quad \forall i \in \mathbb{N},
$$

and a sequence $\delta_{i} \rightarrow 0$ of nonzero reals such that $f=\lim _{i \rightarrow \infty}\left(x_{i}-x\right) / \delta_{i}$ exists. Then

$$
u(f) \in \operatorname{Stab}\left(\pi_{0}(u(x) v)\right) .
$$


Proof. For any sequence $t_{i} \rightarrow \infty$ and $w_{i} \rightarrow w$ in $V^{-}+V^{0}$, we have $a_{t_{i}} w_{i} \rightarrow \pi_{0}(w)$ as $i \rightarrow \infty$. In particular,

$$
a_{t_{i}} u\left(x_{i}\right) v \stackrel{i \rightarrow \infty}{\longrightarrow} \pi_{0}(u(x) v) .
$$

Put $t_{i}=(1 / n) \log \left(\delta_{i}^{-1}\right)$ for all $i$. Then

$$
\begin{aligned}
a_{t_{i}} u\left(x_{i}\right) & =a_{t_{i}} u\left(x_{i}-x\right) u(x) v \\
& =u\left(e^{n t_{i}}\left(x_{i}-x\right)\right)\left(a_{t_{i}} u(x) v\right) \stackrel{i \rightarrow \infty}{\longrightarrow} u(f) \pi_{0}(u(x) v) .
\end{aligned}
$$

Therefore $u(f)$ stabilizes $\pi_{0}(u(x) v)$.

Corollary 4.6. Let $\varphi: I=[a, b] \rightarrow \mathbb{R}^{n-1}$ be a differentiable curve which is not contained in a proper affine subspace of $\mathbb{R}^{n-1}$. Let $0 \neq v \in$ $V$ be such that

$$
u(\varphi(s)) v \in V^{0}+V^{-}, \quad \forall s \in I .
$$

Then $v$ is stabilized by $G$.

Proof. Since $\varphi$ is differentiable and $\varphi(I)$ not contained in a proper affine subspace of $\mathbb{R}^{n-1}$, we conclude that for any $s_{0} \in I$, the set $\mathcal{E}_{0}:=\left\{\varphi(s)-\varphi\left(s_{0}\right): s \in I\right\}$ is not contained in a finite union of proper subspaces of $\mathbb{R}^{n-1}$. Now we can apply Corollary 4.4 to the set $\mathcal{E}=\{\varphi(s): s \in I\}$ and conclude that $\pi_{0}(u(\varphi(s)) v) \neq 0$ is stabilized by $\mathrm{Z}_{\mathrm{G}}(A)$ for all $s \in I$. Now let $s_{0} \in I$ such that $\dot{\varphi}\left(s_{0}\right) \neq 0$. Let $\delta_{i} \rightarrow 0$ be a sequence of of nonzero reals. Then $\dot{\varphi}\left(s_{0}\right)=\lim _{i \rightarrow \infty}\left(\varphi\left(s_{i}\right)-\varphi\left(s_{0}\right)\right) / \delta_{i}$. Therefore by Lemma 4.5 $\pi_{0}\left(u\left(\varphi\left(s_{0}\right)\right) v\right)$ is stabilized by $u\left(\dot{\varphi}\left(s_{0}\right)\right)$. Now the subgroup, say $Q$, generated by $u\left(\dot{\varphi}\left(s_{0}\right)\right)$ and $\mathrm{Z}_{\mathrm{G}}(A)$ contains $\{u(\boldsymbol{x})$ : $\left.\boldsymbol{x} \in \mathbb{R}^{n-1}\right\}$. Therefore $Q$ is a parabolic subgroup of $G$. Since $Q$ stabilizes $\pi_{0}\left(u\left(\varphi\left(s_{0}\right)\right) v\right.$ ), we conclude that $G$ stabilizes $\pi_{0}\left(u\left(\varphi\left(s_{0}\right)\right) v\right)$.

We put $v_{0}=u\left(\varphi\left(s_{0}\right) v\right)-\pi_{0}\left(u\left(\varphi\left(s_{0}\right) v\right)\right)$. Then

$$
u\left(\varphi(s)-\varphi\left(s_{0}\right)\right) v_{0}=u(\varphi(s)) v+\varphi_{0}\left(u\left(\varphi\left(s_{0}\right)\right) v\right) \in V^{-} 0+V^{-}, \quad \forall s \in I .
$$

We choose a finite subset $I_{1} \subset I$ containing $s_{0}$ such that $\{\varphi(s)-$ $\left.\varphi\left(s_{0}\right): s \in I_{1}\right\}$ is an affine basis of $\mathbb{R}^{n-1}$, and apply Proposition 4.2. Therefore if $v_{0} \neq 0$ then $\pi_{0}\left(v_{0}\right) \neq 0$. Since by our choice $\pi_{0}\left(v_{0}\right)=0$, we conclude that $v_{0}=0$. Therefore $u\left(\varphi\left(s_{0}\right)\right) v$ is stabilized by $G$. Hence $v$ is stabilized by $G$, because $u\left(\varphi\left(s_{0}\right)\right) \in G$.

Corollary 4.7. Let $\varphi:[a, b] \rightarrow \mathbb{R}^{n-1}$ be a differentiable curve, whose image is not contained in a proper affine subspace of $\mathbb{R}^{n-1}$. Let $V$ be a finite dimensional normed linear space on which $G$ acts linearly. Further suppose that there is no nonzero $G$-fixed vector in $V$. Then 
given $C>0$ there exists $t_{0}>0$ such that

$$
\sup _{s \in I}\left\|a_{t} u(\varphi(s)) v\right\| \geq C\|v\|, \quad \forall v \in V \text { and } t>t_{0} .
$$

Proof. If the conclusion of the proposition fails to hold then there exists $C>0$ and a sequence $t_{i} \rightarrow \infty$ and convergent sequence $v_{i} \rightarrow v$ in $V$ such that $\|v\|=1$, and

$$
\sup _{s \in I}\left\|a_{t_{i}} u(\varphi(s)) v_{i}\right\| \leq C\left\|v_{i}\right\|, \quad \forall i \in \mathbb{N} .
$$

Therefore we conclude that for any $s \in I$,

$$
\pi_{+}(u(\varphi(s) v))=\lim _{i \rightarrow \infty} \pi_{+}\left(u(\varphi(s)) v_{i}\right)=0 .
$$

In other words,

$$
u(\varphi(s)) v \subset V^{-}+V^{0}, \quad \forall s \in I .
$$

Then by Corollary 4.6, $v$ is fixed by $G$. But this contradicts our hypothesis and the proof is complete.

\section{RATNER'S THEOREM AND DYNAMICAL BEHAVIOUR OF TRANSLATED TRAJECTORIES NEAR SINGULAR SETS}

Our aim is to prove that $\mu$, as obtained in Corollary 3.5, is $L$ invariant. As explained in $\$ 1.1$, we will use a technique from [17].

5.1. Twisted curves and limit measure. Let $\dot{\varphi}(s)$ denotes the tangent to the curve $\varphi$ at $s$. Now onwards we shall assume that $\dot{\varphi}(s) \neq 0$ for all $s \in I$.

Fix $w_{0} \in \mathbb{R}^{n-1} \backslash\{0\}$, and define

$$
W=\left\{u\left(s w_{0}\right): s \in \mathbb{R}\right\} .
$$

Recall that $\mathrm{Z}_{\mathrm{G}}(A)$ acts on $\mathbb{R}^{n-1}$ via the correspondence $u(z \cdot v)=$ $z u(v) z^{-1}$ for all $z \in \mathrm{Z}_{\mathrm{G}}(A)$ and $v \in \mathbb{R}^{n-1}$. This action is transitive on $\mathbb{R}^{n-1} \backslash\{0\}$. Therefore there exists an analytic function $z: I \rightarrow \mathrm{Z}_{\mathrm{G}}(A)$ such that

$$
z(s) \cdot \dot{\varphi}(s)=w_{0} .
$$

For any $i \in \mathbb{N}$, let $\lambda_{i}$ be the probability measure on $L / \Lambda$ defined by

$$
\int_{L / \Lambda} f d \lambda_{i}:=\frac{1}{|I|} \int_{s \in I} f\left(z(s) a_{i} u(\varphi(s)) x_{i}\right) d s, \quad \forall f \in \mathrm{C}_{\mathrm{c}}(L / \Lambda) .
$$

Corollary 5.1. After passing to a subsequence, $\lambda_{i} \rightarrow \lambda$ with respect to the weak-* topology on the space of probability measures on $L / \Lambda$. 
Proof. Given $\epsilon>0$, by Theorem 3.1 there exists a compact set $\mathcal{F} \subset$ $L / \Lambda$ be such that $\mu_{i}(\mathcal{F}) \geq 1-\epsilon$ for all $i>0$. Since $z(I)$ is compact, there exists a compact set $\mathcal{F}_{1} \supset z(I) \mathcal{F}$. Then $\lambda_{i}\left(\mathcal{F}_{1}\right) \geq 1-\epsilon$ for all $i$. Now the corollary is deduced by standard arguments using the one-point compactification of $L / \Lambda$.

\subsection{Invariance under unipotent flow.}

Proposition 5.2. Suppose that $\lambda \stackrel{i \rightarrow \infty}{\longrightarrow} \lambda$ in the space of probability measures on $L / \Lambda$ with respect to the weak-* topology. Then $\lambda$ is $W$ invariant.

Proof. This statement can be deduced by an argument identical to that of the proof of [17, Theorem 3.1]. An idea of this proof is based on the explanation related to (1.10)-(1.12).

Now we shall describe the measure $\lambda$ using Ratner's [13] description of ergodic invariant measures for unipotent flows. Let $\pi: L \rightarrow L / \Lambda$ denote the natural quotient map. Let $W$ be as defined in (5.11). For $H \in \mathscr{H}$, define

$$
\begin{aligned}
N(H, W) & =\left\{g \in G: g^{-1} W g \subset H\right\} \quad \text { and } \\
S(H, W) & =\bigcup_{\substack{F \in \mathscr{H} \\
F \subsetneq H}} N(F, W) .
\end{aligned}
$$

Then by Ratner's theorem [13], as explained in [12, Theorem 2.2]:

Theorem 5.3 (Ratner). Given the $W$-invariant probability measure $\lambda$ on $L / \Lambda$, there exists $H \in \mathscr{H}$ such that

$$
\lambda(\pi(N(H, W))>0 \quad \text { and } \quad \lambda(\pi(S(H, W))=0 .
$$

Moreover almost every $W$-ergodic component of $\lambda$ on $\pi(N(H, W))$ is a measure of the form $g \mu_{H}$, where $g \in N(H, W) \backslash S(H, W)$ and $\mu_{H}$ is a finite $H$-invariant measure on $\pi(H) \cong H / H \cap \Lambda$. In particular if $H$ is a normal subgroup of $L$ then $\lambda$ is $H$-invariant.

5.3. Algebraic criterion for non-accumulation of measure on singular set. Let $\mathscr{A}=\left\{v \in V: v \wedge w_{0}=0 \in \wedge^{d+1} \mathfrak{g}\right\}$. Then $\mathscr{A}$ is the image of a linear subspace of $V$. We observe that

$$
N(H, W)=\left\{g \in L: g \cdot p_{H} \in \mathscr{A}\right\} .
$$

Proposition 5.4 (Cf. [5]). Given a compact set $\mathcal{C} \subset \mathscr{A}$ and $\epsilon>0$, there exists a compact set $\mathcal{D} \subset \mathscr{A}$ containing $\mathcal{C}$ such that given any neighbourhood $\Phi$ of $\mathcal{D}$ in $V$, there exists a neighbourhood $\Psi$ of $\mathcal{C}$ in $V$ contained in $\Phi$ such that for any $h \in G$, any $v \in V$ and any open interval $J \subset I$, one of the following holds: 
I) $h z(t) u(\varphi(t)) v \in \Phi$ for all $t \in J$.

II) $|\{t \in J: h z(t) u(\varphi(t)) v \in \Psi\}| \leq \epsilon|\{t \in J: h z(t) u(\varphi(t)) v \in \Phi\}|$.

Proof. As noted in [17, Proposition 4.4], the argument in the proof of [5. Proposition 4.2] goes through with straightforward changes using the Proposition 3.3 instead of [5, Lemma 4.1].

The next criterion is the main outcome of the linearization technique.

Proposition 5.5 (Cf. [12]). Let $C$ be any compact subset of $N(H, W) \backslash$ $S(H, W)$. Let $\epsilon>0$ be given. Then there exists a compact set $\mathcal{D} \subset \mathscr{A}$ such that given any neighbourhood $\Phi$ of $\mathcal{D}$ in $V$, there exists a neighbourhood $\mathcal{O}$ of $\pi(C)$ in $L / \Lambda$ such that for any $h_{1}, h_{2} \in L$, and a subinterval $J \subset I$, one of the following holds:

a) There exists $\gamma \in \Lambda$ such that $\left(h_{1} z(t) u(\varphi(t)) h_{2} \gamma\right) p_{H} \in \Phi, \forall t \in J$.

b) $\left|\left\{t \in J: h_{1} z(t) u(\varphi(t)) \pi\left(h_{2}\right) \in \mathcal{O}\right\}\right| \leq \epsilon|J|$.

Proof. Again this result and its proof are essentially same as those of [17, Proposition 4.5].

\subsection{Applying the criterion and the basic lemma.}

Theorem 5.6. Suppose that $\lambda_{i} \stackrel{i \rightarrow \infty}{\longrightarrow} \lambda$ in the space of probability measures on $L / \Lambda$ with respect to weak-* topology. Then $\lambda$ is $L$-invariant.

Proof. By Proposition 5.2, $\lambda$ is invariant under the action of the nontrivial unipotent subgroup $W$. Therefore by Theorem 5.3 there exists $H \in \mathscr{H}$ such that

$$
\lambda(\pi(N(H, W))>0 \quad \text { and } \quad \lambda(\pi(S(H, W))=0 .
$$

Let $C$ be a compact subset of $N(H, W) \backslash S(H, W)$ such that $\lambda(C)>\epsilon$ for some $\epsilon>0$. In other words, if we write $x_{0}=\pi\left(g_{0}\right)$ for some $g_{0} \in G$, then there exists a sequence $g_{i} \rightarrow g_{0}$ such that $x_{i}=\pi\left(g_{i}\right)$. Given any neighbourhood $\mathcal{O}$ of $\pi(C)$ in $L / \Lambda$, there exists $i_{0}>0$ such that for all $i \geq i_{0}$, we have $\lambda_{i}(\mathcal{O})>\epsilon$ for all $i>i_{0}$ and hence

$$
\frac{1}{|I|}\left|\left\{s \in I: z(s) a_{t_{i}} u(\varphi(s)) x_{i}=\pi\left(a_{t_{i}} z(s) u(\varphi(s)) g_{i}\right) \in \mathcal{O}\right\}\right|>\epsilon .
$$

Let $\mathcal{D} \subset \mathscr{A}$ be as in the statement of Proposition 5.5. Choose any compact neighbourhood $\Phi$ of $\mathcal{D}$ in $V$. Then there exists a neighbourhood $\mathcal{O}$ of $\pi(C)$ in $L / \Lambda$ such that one of the two possibilities of the Proposition 5.5 holds. Therefore due to (5.7), for all $i>i_{0}$ there exists $\gamma_{i} \in \Lambda$ such that

$$
\left(z(s) a_{t_{i}} u(s) g_{i} \gamma_{i}\right) p_{H}=\left(a_{t_{i}} z(s) u(s) g_{i} \gamma_{i}\right) p_{H} \in \Phi, \quad \forall s \in I .
$$


Let $\Phi_{1}=\left\{z(s)^{-1}: s \in I\right\} \Phi$. Then $\Phi_{1}$ is contained in a compact subset of $V$, and the following holds:

$$
a_{t_{i}} u(s)\left(g_{i} \gamma_{i}\right) p_{H} \subset \Phi_{1}, \quad \forall s \in I, \forall i>i_{0} .
$$

Now we express $V=W_{0} \oplus W_{1}$, where $W_{0}$ is the subspace consisting of all $G$-fixed vectors and $W_{1}$ is its $G$-invariant complement. For $i \in$ $\{0,1\}$, let $P_{i}: V \rightarrow W_{i}$ denote the associated projection. Consider any norm $\|\cdot\|$ on $V$ such that

$$
\|w\|=\max \left\{\left\|P_{0}(w)\right\|,\left\|P_{1}(w)\right\|\right\}, \quad \forall w \in V .
$$

Let $R=\sup \left\{\|w\|: w \in \Phi_{1}\right\}$. By (5.9), for all $i \geq i_{0}$ and $s \in I$ we have (5.11)

$$
\left\|a_{t} u(\varphi(s))\left(g_{i} \gamma_{i} p_{H}\right)\right\|=\left\|P_{0}\left(g_{i} \gamma_{i} p_{H}\right)\right\|+\left\|a_{t} u(\varphi(s)) P_{1}\left(g_{i} \gamma_{i} p_{H}\right)\right\|<R .
$$

Therefore, by Corollary 4.7 applied to $W_{1}$ in place of $V$ and $C=1$, there exists $i_{1}>i_{0}$ such that

$$
\left\|P_{1}\left(g_{i} \gamma_{i} p_{H}\right)\right\|<R, \quad \forall i>i_{1} .
$$

Combining (5.10), (5.11) and (5.12) we have

$$
\left\|g_{i} \gamma_{i} p_{H}\right\|<R, \quad \forall i \geq i_{1} .
$$

The orbit $\Lambda \cdot p_{H}$ is discrete due to Proposition 3.2. And $g_{i} \rightarrow g_{0}$ as $i \rightarrow \infty$. Therefore by passing to a subsequence we may assume that $\gamma_{i} p_{H}=\gamma_{i_{1}} p_{H}$ for all $i \geq i_{1}$. Put $\delta_{0}=\left\|P_{1}\left(g_{0} \gamma_{i_{1}} p_{H}\right)\right\|>0$ and $C=2 R \delta_{0}^{-1}$. Then By Corollary 4.7, there exists $i_{2} \geq i_{1}$ such that for all $i \geq i_{2}$ we have

$$
\sup _{s \in I}\left\|a\left(t_{i}\right) u(\varphi(s)) P_{1}\left(g_{i} \gamma_{i_{1}} p_{H}\right)\right\| \geq C\left\|P_{1}\left(g_{i} \gamma_{i_{1}} p_{H}\right)\right\|>R .
$$

This contradicts (5.11) for all $i \geq i_{2}$, unless $P_{1}\left(g_{0} \gamma_{i_{1}} p_{H}\right)=0$. Hence $g_{0} \gamma_{i_{1}} p_{H}$ is $G$-fixed. Since $\Lambda \cdot p_{H}$ is closed in $V, \Lambda \operatorname{Stab}\left(p_{H}\right)=\Lambda \mathrm{N}_{\mathrm{L}}^{1}(H)$ is closed in $L$. Therefore by taking the inverse $\mathrm{N}_{\mathrm{L}}^{1}(H) \Lambda$ is closed in $L$. Hence the orbit $\pi\left(\mathrm{N}_{\mathrm{L}}^{1}(H)\right)$ is closed in $L / \Lambda$. By [15, Thm. 2.3] there exists a closed subgroup $H_{1}$ of $\mathrm{N}_{\mathrm{L}}^{1}(H)$ of $L$ containing all Ad-unipotent one-parameter subgroups of $L$ contained in $\mathrm{N}_{\mathrm{L}}^{1}(H)$ such that $H_{1} \cap \Lambda$ is a lattice in $H_{1}$ and $\pi\left(H_{1}\right)$ is closed. Now $\rho(G)$ is generated by unipotent one-parameter subgroups. Therefore if we put $F=g_{0} \gamma_{i_{1}} H_{1}\left(g_{0} \gamma_{i_{1}}\right)^{-1}$, then $\rho(G) \subset F$. Also $F x_{0}=g_{0} \gamma_{i_{1}} \pi\left(H_{1}\right)$ is closed and admits a finite $F$-invariant measure. Hence by our hypothesis in the beginning of Section 3, $F=L$. Therefore $L=H_{1} \subset \mathrm{N}_{\mathrm{L}}^{1}(H)$. Therefore, since $N(H, W) \neq 0$, we have $N(H, W)=L$. In particular, $W \subset H$. Thus $H \cap \rho(G)$ is a normal subgroup of $\rho(G)$ containing $W$. Since $\rho(G)$ is a simple Lie group, $\rho(G) \subset H$. Since $H$ is a normal subgroup of $L$ and $\pi(H)$ is a closed orbit with finite $H$-invariant measure, every orbit of 
$H$ on $L / \Lambda$ is closed and admits a finite $H$-invariant measure. Hence by our hypothesis, $H=L$. Now in view of (5.6), by Theorem $5.3 \lambda$ is $H=L$-invariant.

Corollary 5.7. The measure $\mu$ as in the statement of Corollary 3.5 is the unique $L$-invariant probability measure on $L / \Lambda$.

Proof. Since $\varphi$ is analytic, the set of points where $\dot{\varphi}(s)=0$ is finite. Therefore it is enough to prove the theorem separately for each closed subinterval $J$ of $I$, in place of $I$, under the additional hypothesis that $\dot{\varphi}(s) \neq 0$ for all $s \in J$. Since $\varphi$ is analytic, if $J$ is any subinterval of $I$ with nonempty interior, then $\varphi(J)$ is not contained in a proper affine subspace of $\mathbb{R}^{n-1}$. Therefore without loss of generality we assume that $\dot{\varphi}(s) \neq 0$ for all $s \in I$. Let $z(s) \in \mathrm{Z}_{\mathrm{G}}(A)$ be as defined in (5.2). Given $\epsilon>0$ there exists a neighbourhood $O$ of the $e$ in $\mathrm{Z}_{\mathrm{G}}(A)$ such that $|f(z x)-f(x)|<\epsilon$ for all $x \in L / \Lambda$ and $z \in O$. We consider a partition $I=J_{1} \cup \cdots \cup J_{k}$ such that for any $s, s^{\prime} \in J_{j}$, we have $z(s)^{-1} z\left(s^{\prime}\right) \in O$. For each $j \in\{1, \ldots, k\}$, choose $s_{j} \in J_{j}$, and define the function $f_{j}(x)=f\left(z\left(s_{j}\right)^{-1} x\right)$ for all $x \in L / \Lambda$. Then by Theorem 5.6, applied to the interval $J_{j}$ in the place of $I$, there exists $i_{j}>0$ such that for all $i>i_{j}$, we have

$$
\left|\int_{J_{j}} f_{j}\left(z(s) a_{t_{i}} u(\varphi(s)) x_{i}\right) d s-\right| J_{j}\left|\int_{L / \Lambda} f_{j}(x) d \mu_{L}(x)\right| \leq \epsilon\left|J_{j}\right| .
$$

Since $\mu_{L}$ is $\mathrm{Z}_{\mathrm{G}}(A)$-invariant,

$$
\int_{L / \Lambda} f_{j}(x) d \mu_{L}(x)=\int_{L / \Lambda} f(x) d \mu_{L}(x)=: S_{j} .
$$

Now

$$
\begin{aligned}
& \left|\int_{J_{j}} f\left(a_{t_{i}} u(\varphi(s)) x_{i}\right) d s-\right| J_{j}\left|S_{j}\right| \\
& =\left|\int_{J_{j}} f\left(\left(z(s)^{-1} z\left(s_{j}\right)\right) z\left(s_{j}\right)^{-1} z(s) a_{t_{i}} u(\varphi(s)) x_{i}\right) d s-\right| J_{j}\left|S_{j}\right| \\
& \leq\left|\int_{J_{j}} f_{j}\left(z(s) a_{t_{i}} u(\varphi(s)) x_{i}\right) d s-\right| J_{j}\left|S_{j}\right|+\epsilon\left|J_{j}\right| \\
& \leq 2 \epsilon\left|J_{j}\right|,
\end{aligned}
$$

where (5.19) follows from the choice of $O$ and the partition of $I$ into $J_{j}$ 's, and (5.20) follows from (5.15) and (5.16). 
Therefore for any $i \geq \max \left\{i_{1}, \ldots, i_{k}\right\}$, we have

$$
\begin{aligned}
|I| \cdot\left|\int f d \mu_{i}-\int f d \mu_{L}\right| & =\left|\int_{I} f\left(a_{t_{i}} u(\varphi(s)) x_{i}\right) d s-\right| I\left|\int f(x) d \mu_{L}\right| \\
& \leq \sum_{j=1}^{k}\left|\int_{J_{j}} f\left(a_{t_{i}} u(\varphi(s)) x_{i}\right) d s-\right| J_{j}\left|\int f d \mu_{L}\right| \\
& \leq 2 \epsilon \sum_{j=1}^{k}\left|J_{j}\right| \leq 2 \epsilon|I| .
\end{aligned}
$$

This shows that $\mu$ is $L$-invariant.

Proof of Theorem 1.3. By [15, Theorem 2.3] there exists a smallest subgroup $H$ of $L$ containing $\rho(G)$ such that the orbit $H x_{0}$ is closed and admits a finite $H$-invariant measure. Therefore replacing $L$ by $H$ and $\Lambda$ by the stabilizer of $x_{0}$ in $H$, without loss of generality we may assume that $H=L$.

If (1.5) fails to hold then there exist $\epsilon>0$ and a sequence $t_{i} \rightarrow \infty$ such that for each $i$,

$$
\left|\frac{1}{|b-a|} \int_{a}^{b} f\left(\rho\left(a_{t_{i}} u(\varphi(s))\right) x_{0}\right) d s-\int_{L / \Lambda} f d \mu_{L}\right| \geq \epsilon
$$

If we put $x_{i}=x_{0}$ for each $i$, then in view of (3.1) and Corollary 3.5, this statement contradicts Corollary 5.7.

Proof of Theorem 1.6. Note that if the theorem fails to hold then, there exist sequences $x_{i} \rightarrow x_{0}$ in $\mathcal{K}$ and $t_{i} \rightarrow \infty$ in $\mathbb{R}$ such that

$$
\left|\frac{1}{|b-a|} \int_{a}^{b} f\left(a_{t_{i}} u(\varphi(s)) x_{i}\right) d s-\int f d \mu_{G}\right|>\epsilon,
$$

for all $i$. This statement contradicts Corollary 5.7 .

Proof of Theorem 1.5. Let $\psi_{i, j}(s)$ denote the $i, j$-th coordinate of $\boldsymbol{\psi}(s)$ for all $s \in I$. By our hypothesis, the set $\left\{t: \psi_{1,1}(t)=0\right\}$ is finite. Therefore arguing as in the proof of Corollary [5.7, without loss of generality we may assume that $\psi_{1,1}(s) \neq 0$ for all $s \in I:=[a, b]$. Define

$$
\varphi(s)=\left(\frac{\psi_{1,2}(s)}{\psi_{1,1}(s)}, \ldots, \frac{\psi_{1, n}(s)}{\psi_{1,1}(s)}\right) \in \mathbb{R}^{n-1}, \quad \forall s \in I .
$$

Let $U^{-}=\left\{g \in G: a_{t} g a_{t}^{-1} \stackrel{t \rightarrow \infty}{\longrightarrow} e\right\}$. Then there exist continuous curves $\psi_{-}: I \rightarrow U^{-}$and $\psi_{0}: I \rightarrow \mathrm{Z}_{\mathrm{G}}(A)$ such that

$$
\psi(s)=\psi_{-}(s) \psi_{0}(s) u(\varphi(s)), \quad \forall s \in I .
$$


We observe that the curve $\{\varphi(s): s \in I\}$ is contained in a proper affine subspace of $\mathbb{R}^{n-1}$ if and only if the curve $\left.\left\{\left(\psi_{1,1}(s)\right)_{1 \leq j \leq n}\right): s \in I\right\}$ is contained in a proper subspace of $\mathbb{R}^{n}$. Given any $\epsilon>0$ and $f \in$ $\mathrm{C}_{\mathrm{c}}(L / \Lambda)$, there exists $t_{0}>0$ such that for all $t \geq t_{0}$ and $x \in L / \Lambda$, we have $\left|f\left(a_{t} \psi_{-}(s) x\right)-f\left(a_{t} x\right)\right|<\epsilon$. Therefore without loss of generality we may replace $\psi(s)$ by $\psi_{0}(s) u(\varphi(s))$ for all $s \in I$ to prove the theorem.

Now we apply the argument of the proof of Corollary 3.5 to $\psi_{0}(s)$ in place of $z(s)$, and Theorem 1.3 in place of Theorem 5.6, to complete the proof of the theorem.

We shall not provide detailed proofs of other results stated in $\$ 1.2$, as they can be deduced by following the general strategy of [5] and the method of this article.

\section{REFERENCES}

[1] R. C. Baker. Dirichlet's theorem on Diophantine approximation. Math. Proc. Cambridge Philos. Soc., 83(1):37-59, 1978.

[2] Y. Bugeaud. Approximation by algebraic integers and Hausdorff dimension. $J$. London Math. Soc. (2), 65(3):547-559, 2002.

[3] S. G. Dani. Divergent trajectories of flows on homogeneous spaces and Diophantine approximation. J. Reine Angew. Math. 359:55-89, 1985.

[4] S. G. Dani and G. A. Margulis. Asymptotic behaviour of trajectories of unipotent flows on homogeneous spaces. Proc. Indian Acad. Sci. Math. Sci., 101(1):1-17, 1991.

[5] S. G. Dani and G. A. Margulis. Limit distributions of orbits of unipotent flows and values of quadratic forms. In I. M. Gelfand Seminar, pages 91-137. Amer. Math. Soc., Providence, RI, 1993.

[6] H. Davenport and W. M.Schmidt Dirichlet's theorem on diophantine approximation. II. Acta Arith., 16:413-424, 1969/1970.

[7] H. Davenport and W. M. Schmidt. Dirichlet's theorem on diophantine approximation. Symposia Mathematica, Vol. IV (INDAM, Rome, 1968/69), pp. 113-132, Academic Press, London, 1970.

[8] M. M. Dodson, B. P. Rynne, J. A. Vickers. Dirichlet's theorem and Diophantine approximation on manifolds. J. Number Theory, 36(1):85-88, 1990.

[9] Alexander Gorodnik. Open problems in dynamics and related topics. Noted in the workshop on Emerging applications of measure rigidity held at the American Institute of Mathematics in 2004, 2004.

[10] D. Y. Kleinbock and G. A. Margulis. Flows on homogeneous spaces and Diophantine approximation on manifolds. Ann. of Math. (2), 148(1):339-360, 1998.

[11] Dmitry Kleinbock and Barak Weiss. Dirichlet's theorem on Diophantine approximation and homogeneous flows. To appear in Journal of Modern Dynamics. arXiv:math/0612171 1

[12] Shahar Mozes and Nimish A. Shah. On the space of ergodic invariant measures of unipotent flows. Ergodic Theory Dynam. Systems, 15(1):149-159, 1995. 
[13] Marina Ratner. On Raghunathan's measure conjecture. Ann. of Math. (2), 134(3):545-607, 1991.

[14] Marina Ratner. Raghunathan's topological conjecture and distributions of unipotent flows. Duke Math. J., 63(1):235-280, 1991.

[15] Nimish A. Shah. Uniformly distributed orbits of certain flows on homogeneous spaces. Math. Ann., 289(2):315-334, 1991.

[16] Nimish A. Shah. Limit distributions of expanding translates of certain orbits on homogeneous spaces. Proc. Indian Acad. Sci. (Math. Sci.), 106:105-125, 1996.

[17] Nimish A. Shah. Limiting distributions of curves under geodesic flow on hyperbolic manifold. 25 pages. arXiv:0708.4093v1

Tata Institute of Fundamental Research, Mumbai 400005, India

E-mail address: nimish@math.tifr.res.in 